epilepsy, senile dementia, and various other conditions, with gross organic brain changes, inasmuch as in these cases the clinical aspects are chiefly dominated by the intracranial lesions. But of at least 106 cases which during a term of years had come under his care, and which appeared to have some relation to the decision of the question under consideration-the influence of phthisis on the production of insanity, - thirty-four cases were selected as the most reliable. These cases Dr. Mickle placed in five sub-groupsnamely, persons affected with-1. Active, in part quasidelirious symptoms; hallucinations. 2. Depressed, melancholic symptoms. 3. (a) Morose angry mania ; or (b) delusions of injury, of persecution, \&c., not systematised; or (c) rarely gay maniacal excitement (probable organic brain disease). 4. Imperfect or abortive monomania, or monomania. 5. (a) Dementia supervening on feebleness of mind, imbecility ; (b) rarely moral insanity, impulse, and so on. First sub-group: Those in whom mental derangement most clearly and immediately seems to depend on the pulmonary affection are persons forming a comparatively small subgroup, who in advanced phthisis take on active and partly quasi-delirious symptoms. These persons are hallucinated. in most cases certainly, and probably so in the remainder.Second sub-group-the sub-group characterised by mental depression, by symptoms of the melancholic order: Nearly one-third of the cases of the group under consideration come into this sub-group. In all there is emotional depression-downcast, despondent, grieved; some of them are in tears. In very nearly all there are suicidal ideas, and in many these are carried out in suicidal attempts. The emotional state is not always merely one of depression, with or without weeping, moaning, and lugubrious aspect; indefinite fear or dread may possess the patient; he may be afraid to go to sleep, or perhaps vaguely of something about to happen. Some are extremely suspicious; or are timid, anxious, careworn; or are listless; or very obstinate, irritable, unsociable, sullen sulky, slovenly, neglectful, forgetful. Ilallucinations and illusions exist in all, if we include those few cases taking the form of a melancholia cum stupore, where, extremely probable as is their existence, it cannot be definitely ascertained. Relatively predominating are the hallucinations of hearing, next most frequent those of smell, and nearly as many have hallucinations of taste or of sight. At one period or another, or at several, refusal of food is a marked symptom in about two-thirds, and some disinclination for food or anorexia is noted in a few others. Insomnia is a frequent symptom. Some exhibit general apathy, at least at times. A few show marked mental confusion or excitement; and some are inattentive, or mistake the identity of their attendants, taking them for relatives; others wander aimlessly about. Although only slight and transitory in on $\theta$ case, usually of melancholia simplex, delusions are found in all, except in the "thunderstruck" or stuporose, where, too, they doubtless exist. In some they concern the religious feelings and ideas: the patient fancies that he has done some wrong, or is forced to do it by his surroundings, or cut off from hope of heaven, and has anxious, gloomy thoughts of the future. Some take the delusion that they have been poisoned; others fancy themselves victims to the hostility and malevolence of those around them or of absent or imaginary persons. - Third sub-group: Morose, angry mania, or cases with delusions of injury, persecution, not or not yet systematised. Phases and times of emotional depression, though present in some examples, are, Dr. Mickle stated, far less frequent in this than in the previous subgroup, as also are suicidal tendency, apathy, and indolence. On the other hand, many are irritable, sullen, morose, moody; some are obstinate or are given to refuse food; on the other hand, also, excitement predominates - excitement, usually angry, but in some alternately angry and gay; at first, indeed, there may be acute mania. Such patients, too, are usually restless and violent, whether merely destructive or dangerous, or threatening, or homicidal; usually also they are fidgety, gesticulating, muttering, swearing, denouncing; some are noisy, some mentally confused. The memory is often impaired, occupation or amusement unsought, the glance furtive, the visage grimacing.-Fourth sub-group: These cases are of the monomaniacal type, in some instances abortive or imperfect, in some a rapidly deteriorating psychosis. For the most part of the persecutory querulous types, occssionally they are of the hypochondriacal or of the exalted; the characters, however, are often mingled.-Fifth sub-group: In a few cases phthisis appears to widen, deepen, modify the mental defect of a weak-minded person; as it were, to add dementia to a degree of imbecility, and bring the individual affected under notice and into an asylum, owing to the grave loss of mind and the failing mental capacity, then obvious. Such persons are usually young-eighteen to twenty-five. Hopeless as this condition may appear, it is sometimes the theatre of therapeutic triumph, and under outdoor exercise, a regular life, large diet, cod-liver oil and tonics, the patient, may be found to lose the marked signs of phthisis, gain greatly in weight, and, throwing off all the supervenient dementia, return to the degree of mental capacity normal to him, such as it is.--Second group: Cases in which the order of incidence of phthisis and insanity was doubtful-that is to say, in which the two apparently came on simultaneously, or so nearly simultaneously that it could not be definitely stated which of them preceded the other. In many there is emotional depression. This may assume the more common aspect of melancholiz-fear, weeping, grief for alleged (but imaginary) guiltiness of crimes, \&c. But in some the state is apathetic or stuporose, a condition more or less of melancholia attonita. Even still more frequent and striking than phases of emotional depression are the cases with delusions as to bodily injury, damage, or detriment. Sleeplessness is frequent and the great majority are hallucinated, hallucinations of hearing being the most frequent, those of sight next those of other special senses being sometimes observed.Third group: Phthisis supervening on insanity. 1. Relations of forms and clinical aspects of insanity to the subsequent occurrence of phthisis. 2. Relation of phthisis to the modification of pre-existent mental symptoms. 1. Almost any form of mental affection may become complicated by the supervention of phthisis, but some forms, the lecturer stated, are particularly liable thereto. In these latter forms of insanity there are operative, and for a somewhat protracted space of time, not merely such of the general asylum influences as may foster phthisis, but also the chief causes of the mental disease which predispose to phthisisthe special habits, carelessness, and disinclination for or refusal of food. One sub-group, with supervenient phthisis, is that consisting of examples of monomania of the mingled persecutory and expansive form, the persecutory element usually predominating. Another sub-group consists of cases with chiefly unsystematised delusions of persecution, ill-treatment, annoyance, injury, \&c. In $\operatorname{som} \theta$ these delusions, however, tend to become systematised and fixed. In a third sub-group chronic moral and intellectual perversions have followed acute mania, or have supervened on melancholia, now become chronic. Another sub-group consists of stuporose cases, which, whether of the more simple or of the melancholic form, find many phthisical victims. As to the modifications of the mental state wrought by the supervention of phthisis, in about twothirds some change is found. Many patients become more quiet during the phthisis-more depression, less delusion. Dr. Mickle concluded by expressing his regret that time did not allow him to speak of acute tuberculosis and of tubercular meningitis occurring in the insane; of the latency of phthisis in many of them; of the alternations of phthisis and insanity; the salient therapeutic points relating to numerous cases of cure, of prolonged arrest, and vast improvement of phthisis in the insane; the beneficial effect on the temperature of antifebrin, \&c.; and thanked his hearers for their attendance and courtesy.

\section{ON CYCLIC (OR PHYSIOLOGICAL) ALBUMINURIA.}

\section{BX ROBERT BARNES, M.D.}

THE deeply interesting description of "Cyclic Albuminuria" given in THe LANCET of April 14th by Dr, Pavy is full of suggestion for further inquiry. Not many years ago, albuminuria, if at all marked in degree, was looked upon as a condition of almost constant pathological significance. It is now beginning to be recognised that there are varieties of strictly physiological and transient albuminuria. Dr. George Johnson, Dr. Pary, and others, who have done such excellent work in extending our knowledge of this subject, have, however, overlooked the crucial and conclusive evidence in proof of the proposition that albuminuria may exist independently of 
any structural pathological changes in the kidney. This evidence is found in the study of gestation. I have on many occasions (notably in my Lumleian Lectures at the College of Physicians in 1873, and in the "Obstetric Medicine and Surgery" by myself and Fancourt Barnes, 1884.5) enforced the argument that it is impossible to attain to the right appreciation of many of the great problems in general medicine and surgery if the increasing and reverberating light which the careful study of obstetrics can throw upon them be shut out. The problem before us presents a striking illustration.

The theory widely prevailing is that set out by Braun: that albuminuria in pregnancy in a marked and persistent degree is an indication of organic disease of the kidney. This is certainly true in some cases. But it must be remembered that the three stages of Bright's disease described by Braun are revelations of the deadhouse. His descriptions cannot be accepted as true of the cases that completely recover and it is in these cases of recovery that we see direct proof of physiological albuminuria - that is, independent of deep or permanent structural lesion. This truth has been recognised by Depaul, ${ }^{1}$ who expressly says that, in necropsies he had made, the kidneys were perfectly healthy or simply congested. Others have contended that the condition is nephritis, and we must conclude that, as in the case of other organs, inflammation of the kidney may completely pass away. This theory of nephritis was expounded by the illustrious Rayer in 1840, under whom it was my good fortune to study. He called it "néphrite albumineuse." Southey in an excellent clinical lecture ${ }^{2}$ describes the condition under the name "pregnancy nephritis." Other recent authors speak of "acute desquamative nephritis."

Now, I have ventured upon strictly clinical evidence to question the reality of this nephritis as the common explanation. I will not deny its occurrence in exceptional cases; but I do deny that it is frequent, much less general. The problem may, I think, be solved, not by confining our observation to the kidney, but by extending it to the broad field of the phenomena of gestation.

Amongst these correlated phenomena this is what we see: Examining the vaginal-portion of the uterus and the fundus, intense hyperæmia of the mucous membrane, and the surface covered by creamy discharge. This discharge consists of epithelial scales shed under the intense physiological hyperxmia. These scales are in fatty degeneration; they are suspended in an albuminous plasma. A similar condition is often found in other mucous tracts open to direct observation, especially the rectum. It is in the highest degree probable that the mucous membrane of the glandular structure of the kidney, an organ specially within the range of the high vascular tension ruling in gestation, and liable to constant irritation by the waste stuff brought to it for elimination, suffers in like manner. We infer from this that no structural lesion of the kidney is necessary. The kidney conditions attending the initiatory stages of albuminuria and convulsions are simply the result of the high vascular tension, which tells upon the whole mucous tract, and of the attendant bigh nervous tension. This theory is in harmony with the late Dr. Mahomed's observations on the prealbuminuric stage, and with the clinical history of scarlatina, in which disease there is also high nervous and vascular tension, intense congestion of the mucous membrane, and an irritating poison in the blood. This relation seems to have been quite overlooked in the recent discussion in the Obstetrical Society on Scarlatina in Pregnancy and Puerpery.

The following passage from Warburton Begbie is singularly applicable. "What," he asks, "is the cause of the albuminuria in simple scarlstina, and what is its pathological import? I conceive it to be as essential a symptom of the disease as is desquamation of the cuticle-to be, in fact, the result of a desquamative process, which the mucous membranes, equally with the skin, are subject to. Granted, then, that the desquamation occurs when such a change is taking place in the epithelial membrane lining the minute tubes of the kidneys, the office of the cells composing which is to eliminate from the blood the matters, solid or fluid, which in the nor nal exercise of the renal functions compose the urine, it surely is not surprising that the albumen from the former should to a slight amount enter into the latter."

Thus the albuminuria of gestation and the albuminuria

1 L'Union Médicale, 1854

2 The Lancer, 1883. of scarlatina illustrate each other. The exceptional occurrence of albuminuria in both states, and the frequent complete recovery of the kidney in both, exclude the theory of inflammation or Bright's disease as the rule. In both cases it is true that the physiological process is strained to the verge of endurance; in some cases, indeed, the boundary is forced, and physiology yields to pathology. This is more especially the case in scarlatina.

The foregoing discussion throws considerable light upon the history of albuminuria, and especially upon the "cyclic albuminuria" of Dr. Pavy. When physician to the Dreadnought hospital I saw several cases of transient albuminuria from sudden immersion in cold water. Dr. Pavy's observation that albuminuria, absent at other times, appears some little time after the subject has assumed the upright posture, bears further testimony to the influence of relatively high arterial tension.

The general indication for treatment in all the cases of albuminuria, physiological and pathological, is to take off the strain from the kidneys as much as possible, by making the correlated organs-the skin, lung, liver, and intestinesdo full work in elimination, and thus, aided by appropriate agents, as digitalis, to reduce the excessive action of the heart.

\section{ON}

\section{EXTRACTION OF IMMATURE CATARACT.}

\section{B I CHARLES HIGGE NS, F.R.C.S.,} OPHTHALMYO SURGECN TO GUY'S HOSPITAL.

THE treatment of immature nuclear cataract has long been, and still is, one of the vexed questions of ophthalmic surgery. Fxtraction by ordinary methods has not been followed by a good percentage of success on account of the transparent cortex inevitably left behind, which afterwards becomes opaque and blocks the pupil, causes dense capsular obstruction, or sets up severe iritis, leading to closure of the pupil. "Ripening" the cataract by puncturing the lens with a needle, and extracting it when it has become opaque, has been followed by no better results. Washing out the capsule is still under tria!, and in my hands has not been very successful. The method of treatment about to be described has given very fairly good results; the cases reported are the first half-dozen I have done, and the results, although not brilliant, are encouraging. The procedure has for its object the removal of the lens in its capsule. The details of the operation are not quite the same in all the six cases, but I am of opinion that it is best to make the section downwards, and to operate under profound anæsthesia, as follows: A fair-sized iridectomy should be performed downwarde, as a preliminary, some weeks or months before extracting the cataract. I recommend preliminary iridectomy because I believe that the risk of iritis after extraction is much less than if the iris is freshly wounded at the time of extraction. I do it downwards, because the manipulation with the vectis in removal of the cataract is so much more simple when the section is made downwards than when it is made upwards; and also, if the nucleus of the lens alone is opaque, vision may be improved for a time by an artificial pupil in this direction. I remove the lens in its capsule through a small flap section made downwards; the instrument employed for its extraction is the small flexible wire loop, or vectis, designed by Dr. C. B. Taylor of Nottingham. One point comes out clearly in the cases reported-i.e., that the loss of even a considerable quantity of vitreous does not of necessity preclude a fairly successful result. In five out of the six cases vitreous was lost; in one (Case 5) the amount was large. I am of opinion that loss of vitreous does not materially affect the ultimate result of an extraction, provided the whole of the cataract is got away; but if pieces of lens are left mixed up with vitreous upon or behind the iris, severe and very probably destructive iritis will be set up. Case 3, however, would appear to be a contradiction to this view. It should be noticed, in estimating the results obtained in these cases, that in three the eyes were probably unsound; in two (Cases 2 and 3 ) the cataract was associated with high degrees of myopia; and one (Case 6) was an instance of posterior polar cataract-a form 